Central China. At this interview, $68.2 \%$ outpatients were prescribed statins and $24.1 \%$ prescribed doses of statins were sub-minimal.

Conclusion There was a gap between Chinese patients with CHD prevention recommended in guidelines and that in the "real world."

\section{e0298 INTENSIVE CHOLESTEROL LOWERING WITH SIMVASTATIN IMPROVES OUTCOMES OF PERCUTANEOUS CORONARY INTERVENTION IN PATIENTS WITH ACUTE CORONARY SYNDROME}

doi:10.1136/hrt.2010.208967.298

Fu Xianghua, Jia Xinwei, Wang Yanbo, Wang Xuechao, Gu Xinshun, Zhang Jing, Hao Guozhen, Jiang. The Second Hospital of Hebei Medical University

Objective To evaluate the immediate protective effects of intensive statin pretreatment on myocardial perfusion and myocardial ischaemic injury during PCI.

Methods A total of 228 acute coronary syndrome (ACS) patients were randomly divided into standard statin group (SSG, $\mathrm{n}=115$ ) and intensive statin group (ISG, $\mathrm{n}=113$ ). Patients in SSG received $20 \mathrm{mg}$ simvastatin and patients in ISG received 80 mgsimvastatin for 7 days before PCI. TIMI grade flow (TGF), corrected TIMI frame count (CTFC) and TIMI myocardial perfusion grade (TMPG) of the intervened vessel were recorded before and after stent deployment. Plasma level of CK-MB and cTnI were measured before and $24 \mathrm{~h}$ after the procedure.

Results The TFG after stent deployment was significantly improved with less TIMI $0-1$ patients and more TIMI 3 blood flow in ISG than in SSG (all $p<0.05)$. Patients with no reflow phenomenon were less in ISG $(p<0.001)$. The CTFC was lower in ISG than SSG $(p<0.001)$. TMPG was also improved in ISG than SSG $(p=0.001)$. Twenty-four hours after the procedure, although PCI caused significantly increase in CK-MB, the elevated CK-MB value was lower in ISG than SSG $(18.74 \pm 8.41$ vs $21.78 \pm 10.64 \mathrm{p}=0.018)$. Similar changes were also found with regard to Troponin I $(0.99 \pm 1.07$ vs $1.47 \pm 1.54, p=0.006)$. No myocardial infarction was found. Among them, myocardial necrosis was detected in $13 \%$ of the patients in SSG, while $4.4 \%$ in ISG $(p=0.021)$. Myocardial infarction was found in $4.4 \%$ in the patients in SSG and $0.9 \%$ in ISG $(\mathrm{p}=0.213)$.

Conclusion Intensive statin pretreatment for 7 days before PCI can further improve myocardial blood perfusion, protect myocardium from ischaemic injury.

\section{e0299 INCIDENCE AND PREDICTORS OF HYPERTENSION AMONG RURAL CHINESE ADULTS: RESULTS FROM LIAONING PROVINCE}

doi:10.1136/hrt.2010.208967.299

Sun Zhaoging, Sun Yingxian. Shengjing Hospital of China Medical University

Objective To determine the incidence of hypertension and its risk factors among rural Chinese adults.

Methods A population-based sample of 24360 rural Chinese adults aged $\geq 35$ years and free from hypertension at baseline were followed from 2004-2006 to 2008. Incident hypertension was defined as systolic pressure $\geq 140 \mathrm{~mm} \mathrm{Hg}$, diastolic pressure $\geq 90 \mathrm{~mm} \mathrm{Hg}$, or current use of antihypertensive medication.

Results Over a mean of 28 months of follow-up, $29.6 \%$ of men and $23.4 \%$ of women developed hypertension. The age-adjusted incidence rate was higher in men (12.75 per 100 person-years) than in women (10.04 per 100 person-years). Among men, independent predictors of incident hypertension were baseline age $[R R$ per 5 years: 1.11 ; $95 \%$ CI 1.10 to 1.13 ], Mongolian ethnicity (RR: 1.09;
95\% CI 1.01 to 1.18 ), use of alcohol, (RR: $1.14 ; 95 \%$ CI 1.06 to 1.23 ), high income versus low income (RR: $1.11 ; 95 \%$ CI 1.00 to 1.22 ; RR: $1.11 ; 95 \%$ CI 1.03 to 1.20 ), prehypertension versus normotension (RR: 1.18; 95\% CI 1.08 to 1.28), overweight and obesity (RR: 1.28; $95 \%$ CI 1.17 to 1.40 ), baseline salt intake (RR per one g/day: $1.00 ; 95 \%$ CI 1.00 to 1.01 ) and family history of hypertension (RR: 1.14; $95 \%$ CI 1.03 to 1.27). With the exception of use of alcohol, mean income, low physical activity was shown as risk factors in women; the results were similar for women. The awareness, treatment and control rates for newly developed hypertension were 29.9\%, 19.5\% and $1.5 \%$ respectively.

Conclusions These data indicate that the incidence of hypertension is high among these rural Chinese adults and it is associated with many risk factors. And suggest that most newly developed hypertension cases are not treated. These increases in hypertension are probably related to rapid social changes in our country and may apply to other areas of the developing world. These results call for urgent improvements in hypertension prevention and control programs in rural China.

\section{Epidemiology and Preventive Medicine: Lipid Research \\ E0300 RELATIONSHIP OF POLYMORPHISM OF APOLIPOPROTEIN E ALLELES WITH CORONARY HEART DISEASE}

doi:10.1136/hrt.2010.208967.300

Zou Yangchun, Hu Dayi, Tong Qiguang, Xu Liujian, Jia Xingyuan. Beijing Meitan General Hospital

Objective To study the relationship of the apolipoprotein (apo) E with coronary heart disease (CHD).

Methods We studied 68 patients with CHD and 59 control subjects without CHD. Polymorphism of ApoE alleles were measured by PCR, Hhal enzyme digestion and polyacrylamide gel electrophoresis. Results TC, TG, LDL-c, apoB, LP (a) level of CHD group were higher than control $(p<0.05)$; There were significant differences in gene frequency of apo E alleles between CHD and control group but HDL, ApoA1 level of CHD group were lower than control; when compared between the $\mathrm{E} 3$ and the $\mathrm{E} 4$, the $\mathrm{E} 2$ frequency were related with $\mathrm{CHD}(\mathrm{RR}=3.5611,95 \%$ CI 1.1693 to 6.8963, $\mathrm{p}<0.01)$.

Conclusion ApoE gene polymorphisms affects serum TC, LDL-C, apoB and LP (a) levels in CHD and apoE allele E2 is a risk factor in the occurrence of CHD.

\section{e0301 THE RELATIONSHIP BETWEEN APOLIPOPROTEIN E GENE POLYMORPHISMS AND CORONARY ATHEROSCLEROSIS DISEASE IN THE NORTHERN CHINESE}

doi:10.1136/hrt.2010.208967.301

Zou Yangchun, Hu Dayi, Tong Oiguang, Xu Liujian, Jia Xingyuan. Beijing Meitan General Hospital

Background Previous studies on the attribution of apolipoprotein (apo) E polymorphisms on the occurrence of coronary atherosclerosis disease (CAD) showed inconsistent results. Apo $\mathrm{E}$ is a constituent of lipoproteins with considerable variation due to cysteine-arginine exchanges. We investigated the relation between apo E gene polymorphism and the occurrence of $\mathrm{CAD}$ in northern Chinese.

Methods The distributions of the HhaI polymorphisms of the apo $\mathrm{E}$ gene and blood lipids levels were determined among 132 Chinese subjects in relation to circulating lipids and coronary angiography. 